\title{
Chronic insomnia disorder: perspectives from structural neuroimaging
}

\section{Eun Yeon Joo ${ }^{1,2}$}

${ }^{1}$ Department of Neurology, Neuroscience Center, Samsung Biomedical Research Institute, Samsung Medical Center, Sungkyunkwan University School of Medicine, Seoul, Korea

${ }^{2}$ Department of Health Sciences and Technology, Samsung Advanced Institute of Health Sciences and Technology (SAIHST), Sungkyunkwan University, Seoul, Korea

Received: October 23, 2018

Revised: November 20, 2018

Accepted: November 21, 2018

Corresponding author:

Eun Yeon Joo

Department of Neurology,

Samsung Medical Center,

Sungkyunkwan University

School of Medicine, 81 Irwon-ro,

Gangnam-gu, Seoul 06351, Korea

Tel: +82-2-3410-3597

E-mail: ejoo@skku.edu;

eunyeon1220.joo@samsung.com

\begin{abstract}
Chronic insomnia disorder is the most widely reported clinical condition in medicine. It has a significant impact on populations and is characterized by chronically disturbed sleep and sleep loss, non-refreshing sleep, and heightened arousal in bed. Poor sleep is associated with a wide range of negative health outcomes, and it is reported that poorer quality of life and medical, neurological, and psychiatric comorbidities disrupt sleep. Sleep difficulties may result from multiple etiologies; however, the neurobiological mechanisms underlying chronic insomnia disorder are not sufficiently understood. Recently, numerous neuroimaging studies have been conducted to investigate the structural or functional derangement in the brains of patients with chronic insomnia disorder. The development of neuroimaging techniques has provided insight into the pathophysiological mechanisms that make patients with chronic sleep disturbances vulnerable to cognitive impairment.
\end{abstract}

Keywords: Cerebral cortex; Hippocampus; Magnetic resonance imaging; Sleep; Sleep initiation and maintenance disorders

\section{INTRODUCTION}

Chronic insomnia disorder (CID), a clinical condition, is characterized by the subjective experience of chronically disturbed sleep and sleep loss, and there is evidence of conditioned sleep difficulties and/or heightened arousal in bed [1]. These patients often present with difficulties falling asleep and maintaining sleep, and experience significant daytime consequences such as fatigue, sleepiness, and poor psychosocial function. In addition, patients with CID commonly have cognitive impairments and deficits in memory consolidation during sleep compared to that in good sleepers [2,3]. However, the neuroanatomical correlates of these symptoms and signs have not been clearly elucidated. Recent magnetic resonance imaging (MRI) studies providing a unique in vivo assessment of brain structural integrity have suggested that CID is associated with cortical and subcortical morphology alteration, which allow for an explanation of the characteristics of CID [4-9].
This is an Open Access article distributed under the terms of the Creative Commons Attribution Non-Commercial License (http:// creativecommons.org/licenses/ by-nc/4.0/). 
Brain morphometry using high resolution T1-weighted MRI has revealed anatomical brain changes associated with insomnia. Previous studies have considered the hippocampus as an important target of pathogenesis in CID [6,10,11]. A negative correlation between cognitive decline and hippocampal atrophy was reported in CID patients [9]. The frontal lobe and cingulate gyrus have been morphometrically evaluated in chronic insomnia [12]. Nevertheless, inhomogeneous patient brain compositions and different parameters and technologies had yielded inconsistent results across the studies. The current article summarizes the significant findings that localize cortical and subcortical changes associated with the characteristics of CID.

\section{EXPLORING THE CORTEX}

It is well-known that structural imaging may identify the anatomical substrates underlying disease-specific symptoms and signs in the field of neurology. Although MRI scans of individual patients with CID appear normal upon visual inspection, group analyses using diverse imaging techniques may reveal the specific changes in the brains. A number of morphometric studies were performed in insomnia patients using conventional high-resolution T1-weighted magnetic resonance images to quantify the size of specific brain structures. Among numerous methodologies, voxel-based morphometry (VBM) is the most popular automated technique as it provides a comprehensive assessment of anatomical differences throughout the brain $[13,14]$. The first optimized VBM study revealed that patients with CID had gray matter deficits in the left orbitofrontal cortex and precuneus compared to that of controls [15]. Optimized VBM has a significant circularity problem since the registration requires an initial tissue classification and vice versa [16]. In contrast, Statistical Parametric Mapping 8 (SPM8)-based VBM has updated a registration method termed Diffeomorphic Anatomical Registration Through Exponentiated Lie algebra, which is a more sensitive means of identifying differences in gray matter and white matter [16]. Thus, SPM8-based VBM provides more accurate localization than does optimized VBM in terms of supporting precise intersubject alignment and segmentation performance throughout the iterative unified model $[16,17]$.

The first VBM study reported smaller gray matter volumes in the left orbitofrontal and parietal cortices in insomnia patients and a negative correlation between the orbitofrontal gray matter and insomnia severity, without any correlation with mood ratings [15]. A subsequent study [18] reported an increased volume of the rostral anterior cingulate cortex using FreeSurfer (Athinoula A. Martinos Center for Biomedical Imaging; http://www.freesurfer.net), an automated program for measuring the volume of brain structures [19]. However, this finding was not duplicated by one study using both the same methodology and VBM [6], and by two other studies using VBM $[7,15]$. Another study using the SPM8-based VBM method observed a significant reduction of the gray matter concentration in the left and right dorsolateral prefrontal cortices, pericentral cortex, and superior temporal gyrus compared to that in controls with a cluster threshold of 100 voxels at the level of uncorrected $\mathrm{P}<0.001$. When a cluster threshold of more than 50 voxels was applied, the gray matter concentration was found to be decreased in the larger brain areas with the same anatomical coordinates as the results of the cluster threshold of 100 voxels, and was also observed to be decreased in the medial frontal gyri and cerebellum (Fig. 1) [5]. Gray matter deficits in the dorsolateral prefrontal cortex were related to attention deficit, frontal lobe dysfunction, and nonverbal memory decline in patients with CID, which might be associated with poorer sleep. Moreover, reduced gray matter concentrations in the left or right frontal cortices were significantly related to insomnia severity, longer sleep latency, or longer duration of wakefulness after sleep onset (WASO) (Fig. 2) [5]. This finding suggests that disturbed nocturnal sleep has a harmful effect on the frontal cortex of CID patients.

A previous VBM study identified the areas exhibiting only gray matter volume reduction in patients with chronic insomnia [15]. Thus, the two previous studies observed decreases in both gray matter concentrations and volumes, and the brain regions exhibiting volume changes were much smaller than those exhibiting concentration reduction. In optimized VBM, gray matter concentration in the local unit (i.e., voxel) can be transformed to the gray matter volume through the commonly known "modulation" process while accounting for regional stretching and compression occurring during coregistration [20]. The gray matter concentration is typically interpreted as gray matter tissue density relative to white matter, whereas gray matter volume is interpreted as absolute volume regardless of white matter [21]. Quantifying these two measures does not necessarily lead to overlapping results due to their different underlying properties but rather complements aspects of brain structural alterations [20].

Although it is unclear whether gray matter reductions are a preexisting abnormality or a consequence of insomnia, gray 

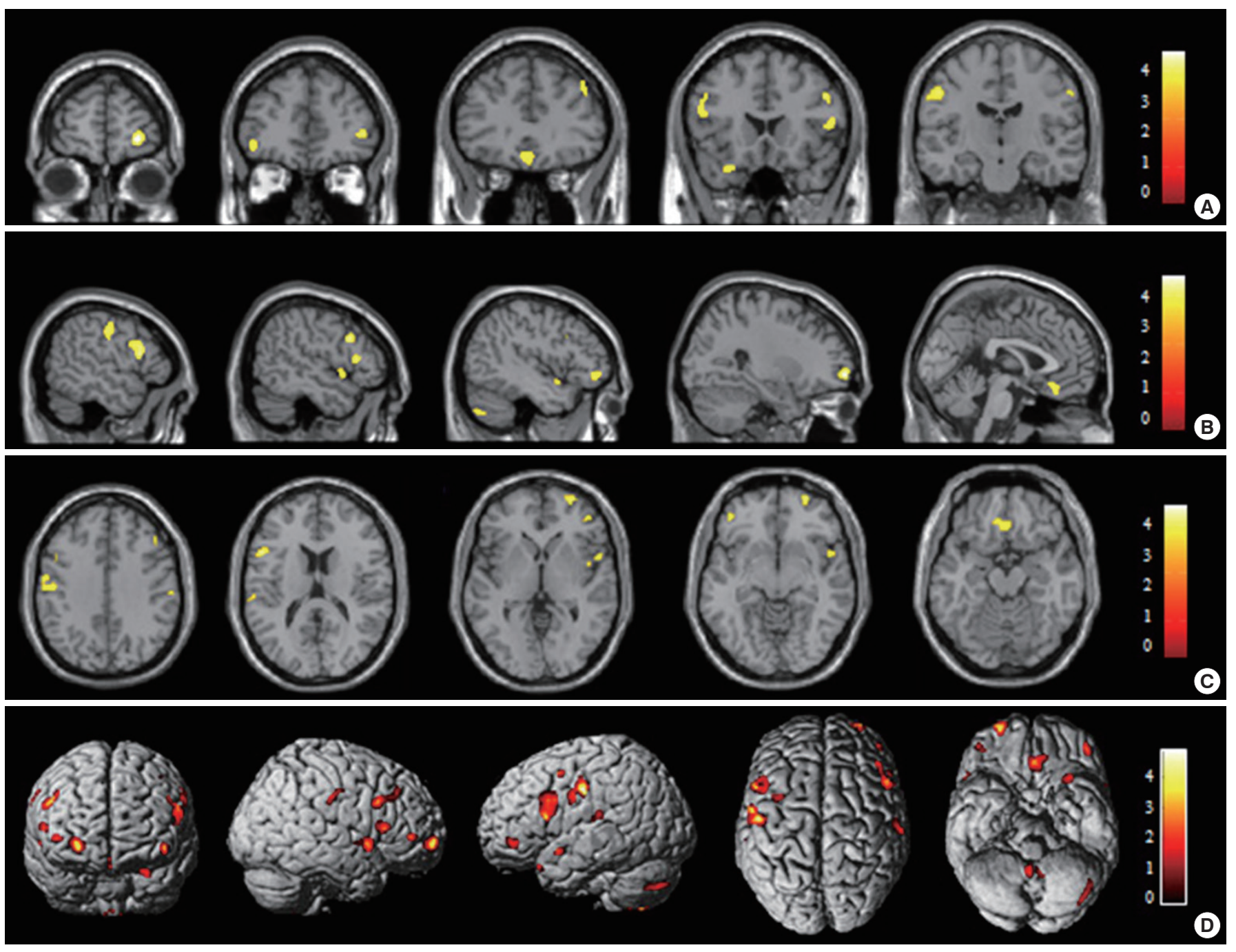

Fig. 1. Voxel-based morphometry indicating a decrease in the gray matter concentration (GMC) in patients with chronic insomnia disorder (CID) compared to that in healthy controls. (A) The GMC was significantly decreased in CID patients (uncorrected at $\mathrm{P}<0.001$, two-sample t-test) in the right superior frontal gyrus, left orbitofrontal gyrus, right inferior frontal gyrus, right medial frontal gyrus, right middle frontal gyrus, right precentral gyrus, and left postcentral gyrus coronal view; (B) left postcentral gyrus, left inferior frontal gyrus, right middle frontal gyrus, right inferior frontal gyrus, right superior temporal gyrus, left middle frontal gyrus, left cerebellum, right superior frontal gyrus, and left medial frontal gyus sagittal view; and (C) left postcentral gyrus, right middle frontal gyrus, right precentral gyrus, left inferior frontal gyrus, right superior frontal gyrus, right inferior frontal gyrus, right superior temporal gyrus, left middle frontal gyrus, and left medial frontal gyrus axial view. (D) The overall areas with reduced GMCs are indicated in a three-dimensional brain surface rendering view. The results were displayed with a cluster threshold of $>50$ voxels. The scales in the color bar are $t$ scores. The left side of the images represents the left hemisphere of the brain. Adapted from Joo et al., with permission from Oxford University Press [5].

matter reduction as well as a lack of sleep or poorer sleep quality in the patients with insomnia might be responsible for the clinical features and cognitive dysfunction in chronic insomnia.

There has been an increase in the number of studies examining the corticocortical structural covariation network that is derived from correlational analysis of morphometrics between multiple cerebral regions [22-25]. Structural covariance may indicate altered neural connectivity resulting from rela- tively long-term processes such as neurodevelopment [26] or neurodegeneration [27]. Analyses of cortical thickness indeed demonstrated structural covariance within regions of the default mode network (DMN) with healthy aging [28]. In 60 selected patients with persistent insomnia symptoms from a population-based cohort study, significant cortical thinning was observed in both hemispheres of patients with insomnia compared to that in controls (left: $2.61 \pm 0.13 \mathrm{~mm}$ vs. $2.66 \pm$ $0.11 \mathrm{~mm}, \mathrm{P}<0.05$; right: $2.60 \pm 0.14 \mathrm{~mm}$ vs. $2.67 \pm 0.12 \mathrm{~mm}$, 
Insomnia severity index with GMCs in left middle frontal gyrus
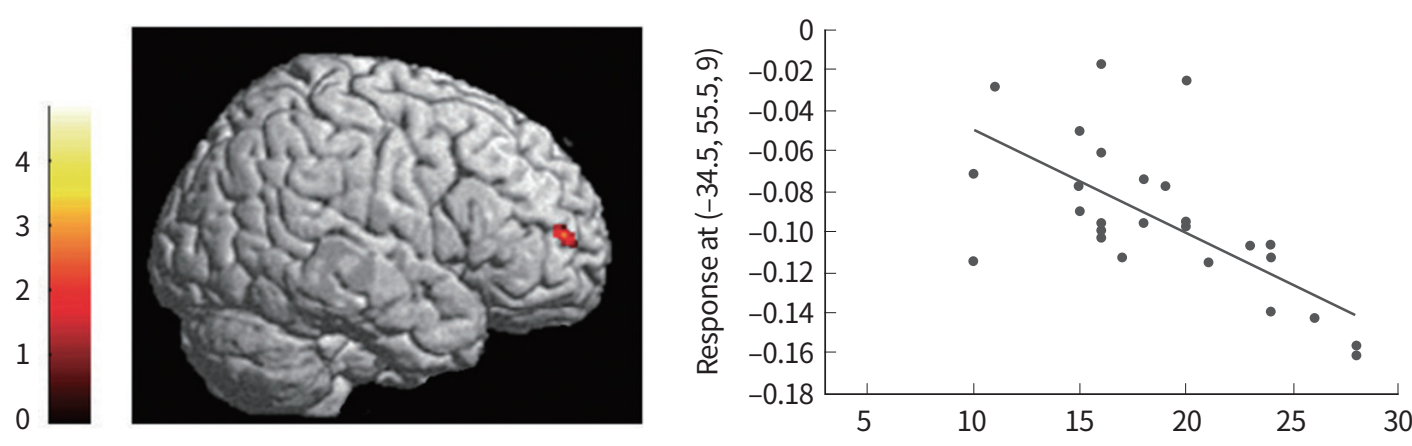

Sleep latency with GMCs in right postcentral gyrus
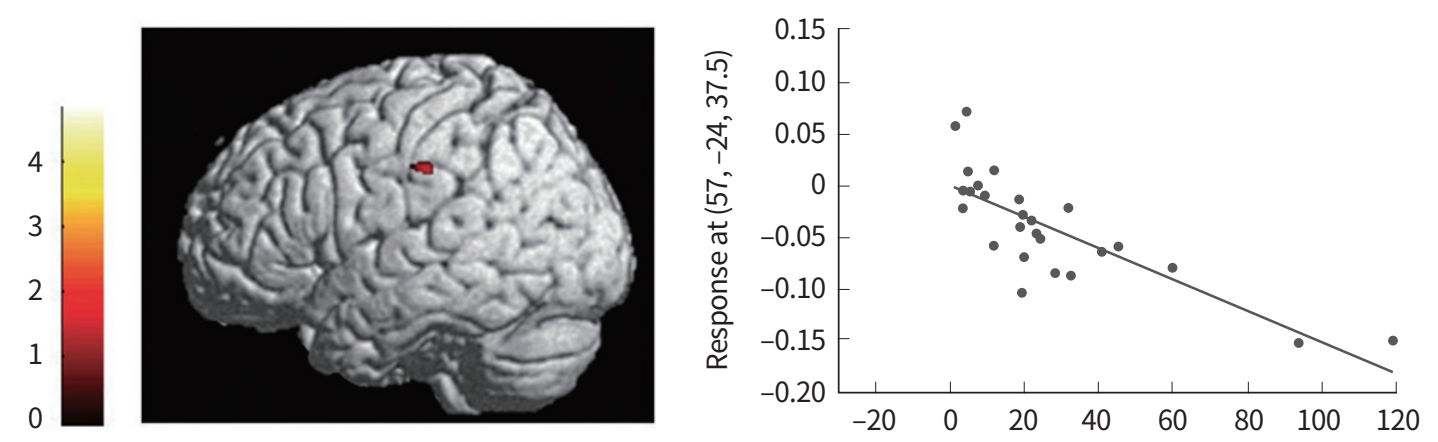

Wakefulness after sleep onset with GMCs in right precentral gyrus
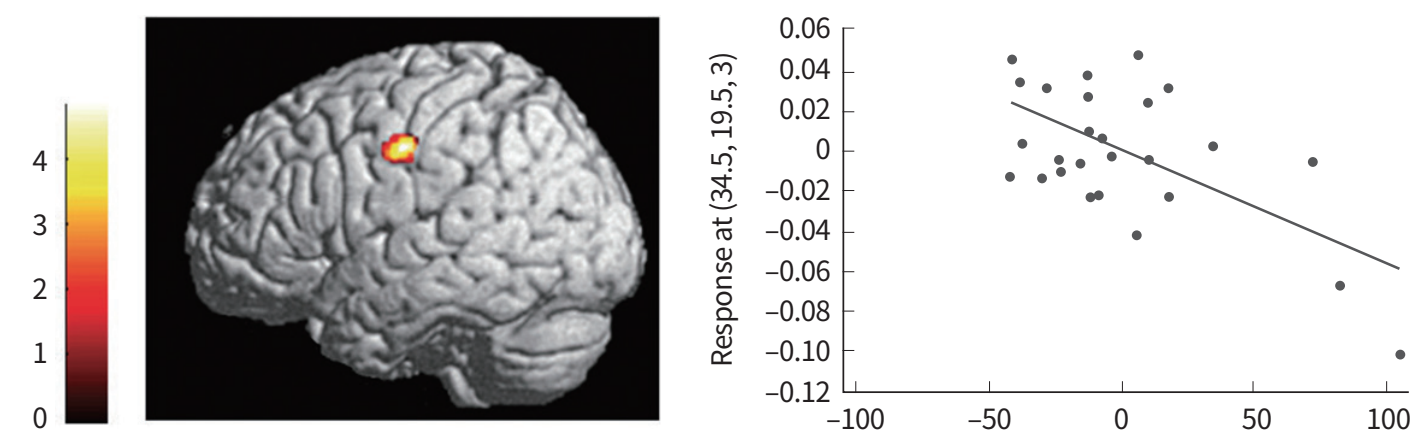

Fig. 2. Correlation between brain cortical regions with the characteristics of patients with chronic primary insomnia. (A) A negative correlation was observed between the gray matter concentration (GMC) in the left middle frontal gyrus and the Insomnia Severity Index $(r=-0.613, P=0.014)$, (B) between the GMC in the right postcentral gyrus and the sleep latency ( $r=-0.411, P=0.019)$, and (C) between the GMC in the right precentral gyrus and wakefulness after sleep onset $(r=-0.443, P=0.018)$. The confounding factors of age, sex, and intracranial volume were controlled. Adapted from Joo et al., with permission from Oxford University Press [5].

$\mathrm{P}<0.01$ ) [8]. Regional analyses revealed that cortical thinning was circumscribed to the left medial frontal cortex, bilateral precentral cortices, and right lateral prefrontal cortex, which was a similar pattern of gray matter decreases as that in the VBM study. Additional analysis of corticocortical morphological covariance demonstrated that insomnia patients only displayed a significant correlation of the medial frontal cortex with most of the frontal cortices, while high covariance $(r>0.5$, false discovery rate $[F D R]<0.001$ ) with the medial frontal cortex seed including the prefrontal cortex, precuneus, and later- al parietal cortex as well as scattered clusters in the temporal lobe was observed in controls. This analysis revealed that morphological alterations in the persistent insomnia group were circumscribed to multiple cortices, primarily the frontal and parietal cortices, and structural covariance was disrupted in the link between these two cortices in insomnia patients. This seems to be the most comprehensive structural imaging study of insomnia, demonstrating anatomical alterations and disrupted structural connectivity, as well as the implications on cognitive function and sleep quality. This study suggests 
that patients with persistent insomnia symptoms present with altered structural connectivity mainly within regions of the DMN that reduces their capacity to perform the normal transition to sleep, accompanied by a functional disconnection between the anterior and posterior regions of the DMN. This altered connectivity may further contribute to the sustained sleep difficulties and cognitive impairment commonly reported by insomnia patients [8].

\section{EXPLORING THE SUBCORTICAL STRUC- TURE}

The negative association of disturbed sleep with performance on memory tasks $[2,29,30]$ implicates hippocampal dysfunction in patients. Thus, there have been controversial findings concerning hippocampal volume in human insomnia studies. A previous pilot study found that the bilateral hippocampal volume was significantly lower in CID patients than in good sleepers [11]. In contrast, a recent study did not find any objective differences in the hippocampal volumes of CID patients, although some patients with sleep maintenance problems were found to have smaller hippocampal volumes, as determined by wrist actigraphy [18]. Although the authors found a significant correlation between reduced volumes in the bilateral hippocampi and poor sleep efficiency and increased WASO in those CID patients. The most recent study [4] reported that CID patients did not exhibit any definitive differences in intracranial volumes or in absolute and intracranial volumes to normalized hippocampal volumes compared to controls. However, a significant correlation was noted between the bilateral hippocampal volume and the duration of insomnia (left: $r=-0.872, P<0.001$; right: $r=-0.868, P<0.001)$ and the arousal index of polysomnography (left: $r=-0.435, P=0.045$; right: $r=-0.409, P=0.026$ ) in patients [4]. In addition, they exhibited significantly impaired attention, frontal lobe function, and memory, and their verbal and nonverbal memory scores were positively correlated with the hippocampal volume. Another study did not reveal any statistical differences in hippocampal volumes between patients and controls [6]. These conflicting results regarding hippocampal volume may be related to the different anatomical landmarks to delineate the boundary of the hippocampus and different subsets of patients examined in the studies [4]. In particular, we observed that the left and right hippocampal volumes in insomnia patients were significantly and negatively correlated with the duration of insomnia and suggested that a longer duration of insomnia might neg- atively influence hippocampal function and volumes. These methodological or demographic inconsistencies always exist in neuroimaging studies, and well-standardized study protocols are required in multicenter trials to clarify the results.

Earlier hippocampal volumetry studies utilized manual delineation of the hippocampal boundary, which underlined the technical limitations of the low sensitivity of global hippocampal volumetry and the variability of hippocampal segmentation. To overcome these issues, automated subfield volumetry was developed and applied in the next study [7]. Vertex (= point)-wise morphometry [31-33] based on a surface extracted from the manual segmentation of the whole hippocampus has been a surrogate to manual subfield volumetry. In 27 CID patients, a significant decrease was observed in the hippocampal volume compared to controls (left: $2,980 \pm 283 \mathrm{~mm}^{3}$ vs. 3,197 $\pm 337 \mathrm{~mm}^{3}$; right: 3,079 \pm 298 $\mathrm{mm}^{3}$ vs. $3,247 \pm 404 \mathrm{~mm}^{3}, \mathrm{P}<0.05$ ) [7]. This change was hemispherically symmetric; asymmetry between patients and controls did not differ. This finding was line with those of the manual hippocampal volumetry studies $[15,18]$. In patients, hippocampal atrophy was identified in all subfields. The largest cluster of atrophy was detected at the level of the hippocampal body and tail (FDR $<0.005,200$ vertices) and was located medially, mainly within the region corresponding to the combined region of cornu ammonis (CA)2-4 and the dentate gyrus (DG) (Fig. 3A) [7]. Atrophy at the level of the head was present on the superomedial surface corresponding to the CA1 region (FDR $<0.05$, 58 vertices). Hippocampal subfield atrophy in CID suggests reduced neurogenesis in the DG and neuronal loss in CA subfields in conditions of sleep fragmentation and the related chronic stress condition of insomnia. Atrophy in the CA3-4-DG region was associated with impaired cognitive function in patients (Fig. 3B), and these observations suggest that patients with chronic sleep disturbance are vulnerable to cognitive impairment.

A recent study exploring the morphological changes in subcortical structures demonstrated that local shape changes in the putamen were associated with higher arousal indices of polysomnography in CID [9]. In patients, atrophic changes in the hippocampus were associated with delayed correct response times in a Stroop word test, decreased phonemic word fluency in a Controlled Oral Word Association Test (COWAT), and lower Korean California Verbal Test (KCVLT) total scores. Amygdala atrophy was correlated with lower KCVLT short-delay free recall and recognition. Moreover, shape analysis of subcortical structures revealed that that lower sleep quality and a higher arousal index were associated with a greater 


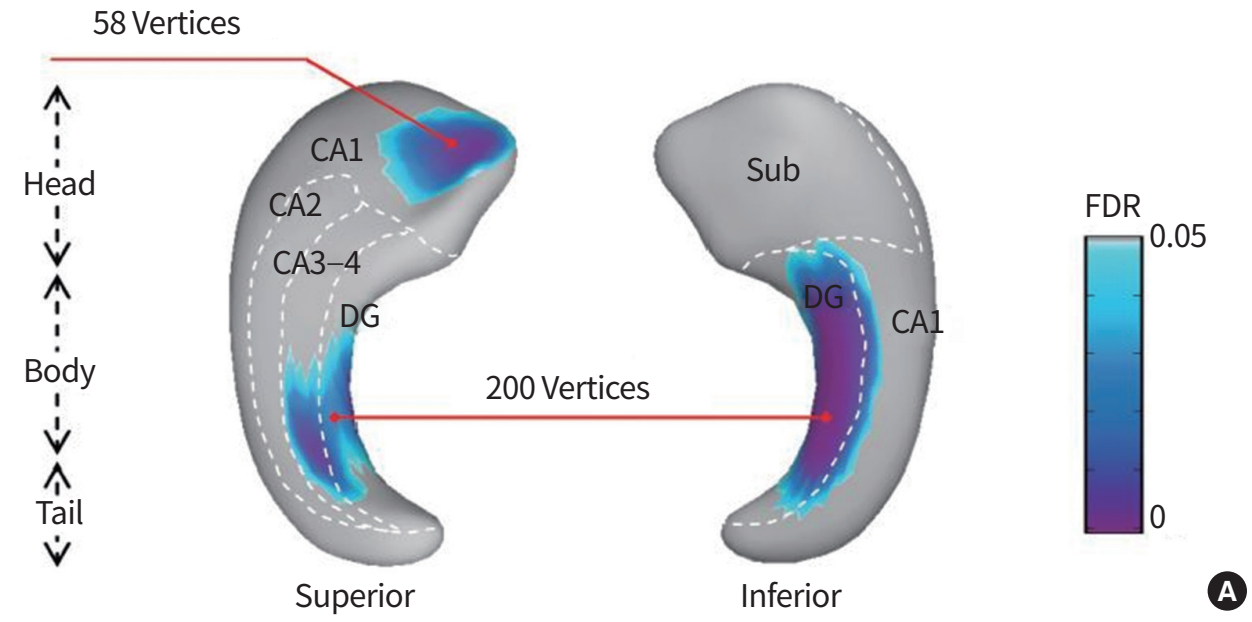

Clinical parameters
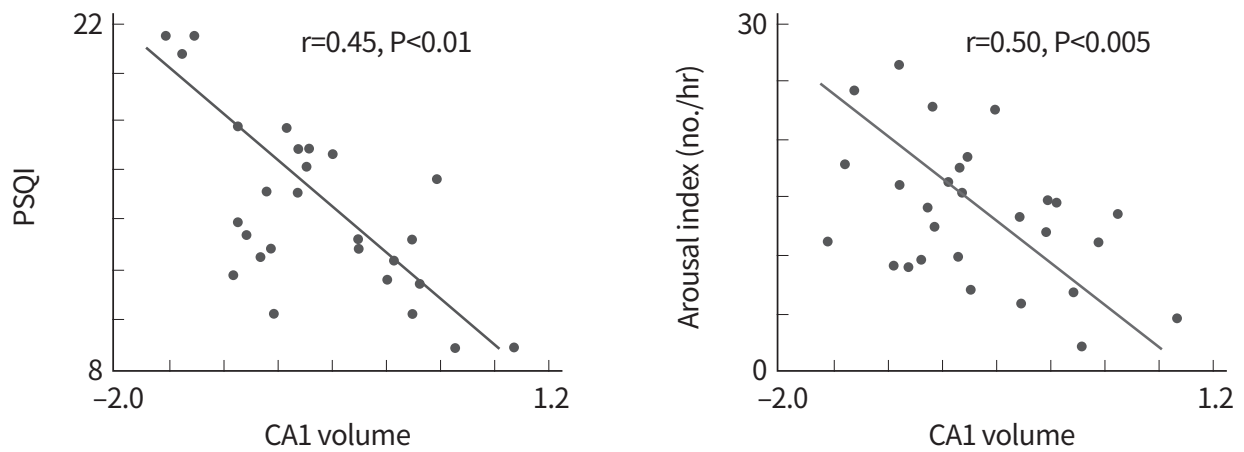

Neuropsychological parameters (in z-score)
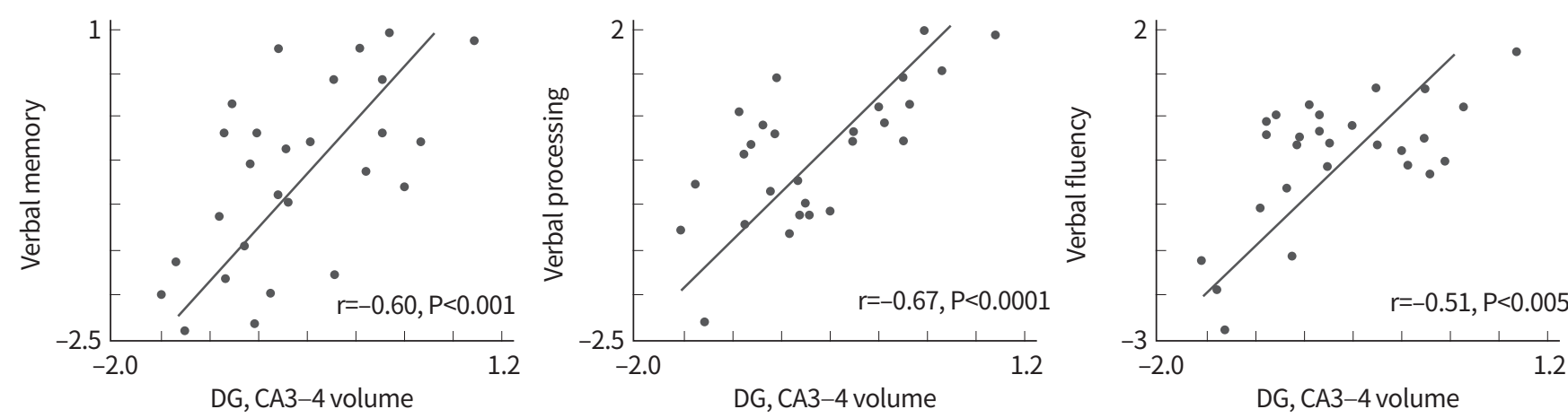

Fig. 3. Vertex-wise group comparison between patients with chronic insomnia disorder and healthy controls. (A) Regions of volume decrease in patients relative to controls are presented. The significance threshold was set at false discovery rate $<0.059$. (B) A negative association was found between the hippocampal subfield volume and clinical (upper) and neuropsychological (lower) parameters in patients with chronic insomnia disorder. For each cluster representing significant volume loss in patients relative to controls, its mean volume is correlated with a given clinical or neuropsychological parameter while controlling for age, sex, and depressive mood. Linear regression models were plotted for significant correlations. Adapted from Joo et al., with permission from Oxford University Press [7]. CA, cornu ammonis; DG, dentate gyrus; FDR, false discovery rate; PSQI, Pittsburgh Sleep Quality Index.

number of atrophic changes in the hippocampus and putamen, and atrophic changes in the basal ganglia and thalamus were related to cognitive decline in neuropsychological domains (Fig. 4). These findings suggested that sleep disturbances in individuals with chronic insomnia are related to cognitive im- pairment consistent with the alteration of frontal-subcortical circuits. Additionally, this surface-based shape analysis method is useful in the localization of subcortical changes that are associated with cognitive decline in patients with CID. 


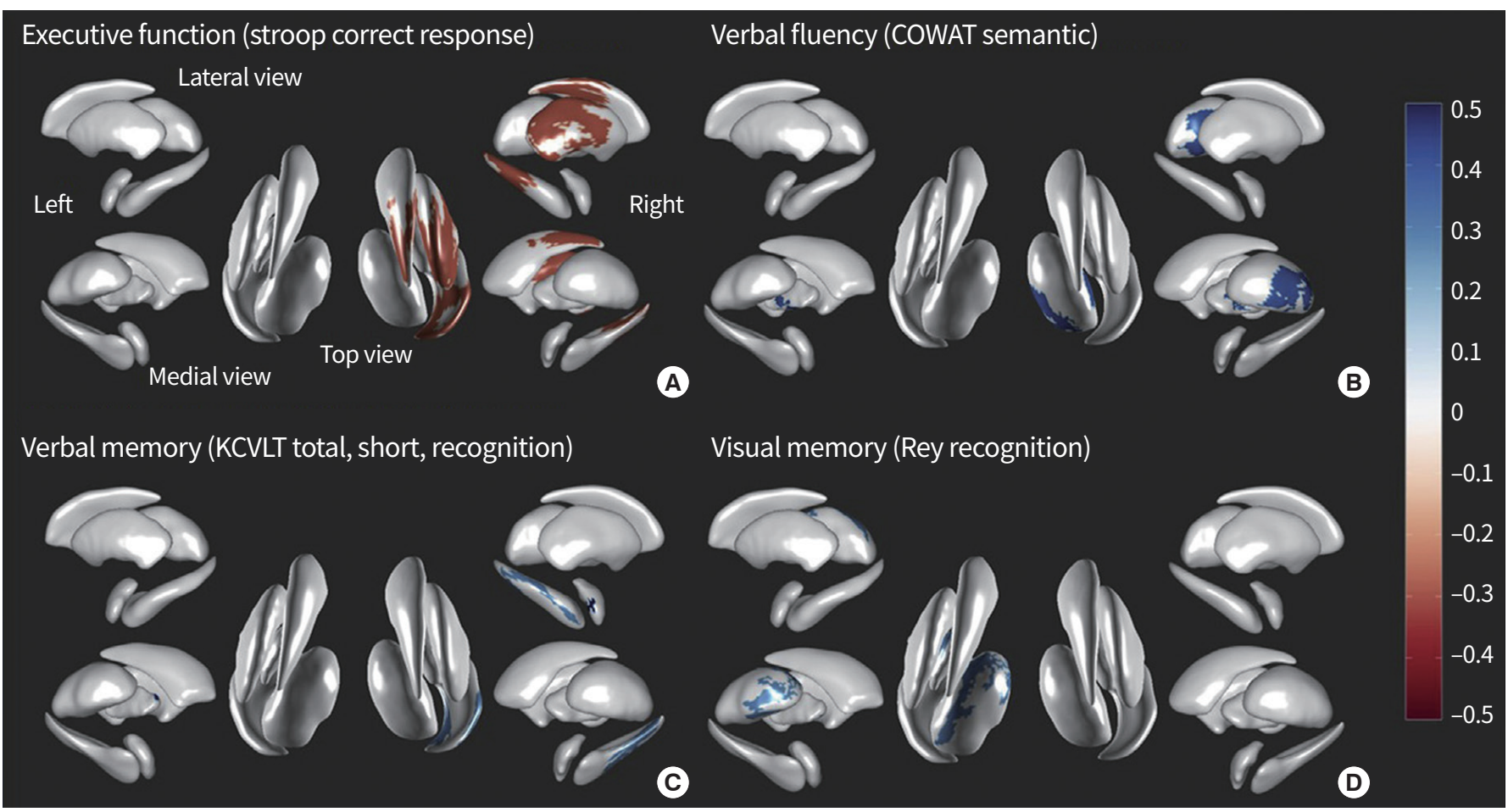

Fig. 4. Subcortical shape changes and cognitive decline in patients with chronic insomnia disorder. Subcortical morphological changes are associated with the impairment of various neuropsychological domains as follows: (A) executive function (Stroop word correct response time); (B) verbal fluency (Controlled Oral Word Association Test [COWAT] semantic word fluency); (C) verbal memory (Korean California Verbal Test [KCVLT] total, short delay free recall, and recognition); and (D) visual memory (Rey Complex Figure Test [RCFT] recognition) (random field theory-corrected $\mathrm{P}<0.05$ ). Adapted from Koo et al., with permission from Elsevier [9].

\section{CONCLUSION}

Sophisticated neuroimaging techniques allow for the in vivo visualization of human brain anatomy with exquisite detail and the quantification of morphological changes. Comprehensive structural imaging studies demonstrated anatomical alterations in the frontal cortex, hippocampus, temporal cortex, or cingulate, and disrupted structural connectivity explaining the cognitive dysfunction and poor sleep quality of CID. Additionally, volumetry and subfield shape analysis identified atrophic changes in the hippocampus and putamen, which provided evidence for the pathophysiological mechanisms underlying the susceptibility of patients with CID to cognitive impairment. Advancements in imaging technology and software in larger, longitudinal studies may enable us to better understand CID and related disorders.

\section{CONFLICTS OF INTEREST}

No potential conflict of interest relevant to this article was reported.

\section{ACKNOWLEDGMENTS}

This research was supported by the Basic Science Research Program through the National Research Foundation of Korea funded by the Ministry of Science, ICT \& Future Planning, Republic of Korea (2017R1A2B4003120) and by a Samsung Biomedical Research Institute grant (SMX1170571).

\section{ORCID}

Eun Yeon Joo https://orcid.org/0000-0003-1233-959X

\section{REFERENCES}

1. Roth T, Roehrs T, Pies R. Insomnia: pathophysiology and implications for treatment. Sleep Med Rev 2007;11:71-9.

2. Backhaus J, Junghanns K, Born J, Hohaus K, Faasch F, Hohagen F. Impaired declarative memory consolidation during sleep in patients with primary insomnia: influence of sleep architecture and nocturnal cortisol release. Biol Psychiatry 2006;60:1324-30.

3. Fulda S, Schulz H. Cognitive dysfunction in sleep disor- 
ders. Sleep Med Rev 2001;5:423-45.

4. Noh HJ, Joo EY, Kim ST, Yoon SM, Koo DL, Kim D, et al. The relationship between hippocampal volume and cognition in patients with chronic primary insomnia. J Clin Neurol 2012;8:130-8.

5. Joo EY, Noh HJ, Kim JS, Koo DL, Kim D, Hwang KJ, et al. Brain gray matter deficits in patients with chronic primary insomnia. Sleep 2013;36:999-1007.

6. Spiegelhalder K, Regen W, Baglioni C, Kloppel S, Abdulkadir A, Hennig J, et al. Insomnia does not appear to be associated with substantial structural brain changes. Sleep 2013;36:731-7.

7. Joo EY, Kim H, Suh S, Hong SB. Hippocampal substructural vulnerability to sleep disturbance and cognitive impairment in patients with chronic primary insomnia: magnetic resonance imaging morphometry. Sleep 2014;37:118998.

8. Suh S, Kim H, Dang-Vu TT, Joo E, Shin C. Cortical thinning and altered cortico-cortical structural covariance of the default mode network in patients with persistent insomnia symptoms. Sleep 2016;39:161-71.

9. Koo DL, Shin JH, Lim JS, Seong JK, Joo EY. Changes in subcortical shape and cognitive function in patients with chronic insomnia. Sleep Med 2017;35:23-6.

10. Neylan TC, Mueller SG, Wang Z, Metzler TJ, Lenoci M, Truran $D$, et al. Insomnia severity is associated with a decreased volume of the CA3/dentate gyrus hippocampal subfield. Biol Psychiatry 2010;68:494-6.

11. Riemann D, Voderholzer U, Spiegelhalder K, Hornyak M, Buysse DJ, Nissen C, et al. Chronic insomnia and MRI-measured hippocampal volumes: a pilot study. Sleep 2007;30: 955-8.

12. O'Byrne JN, Berman Rosa M, Gouin JP, Dang-Vu TT. Neuroimaging findings in primary insomnia. Pathol Biol (Paris) 2014;62:262-9.

13. Wright IC, McGuire PK, Poline JB, Travere JM, Murray RM, Frith $C D$, et al. A voxel-based method for the statistical analysis of gray and white matter density applied to schizophrenia. Neuroimage 1995;2:244-52.

14. Ashburner J, Friston KJ. Voxel-based morphometry: the methods. Neuroimage 2000;11(6 Pt 1):805-21.

15. Altena E, Vrenken H, Van Der Werf YD, van den Heuvel OA, Van Someren EJ. Reduced orbitofrontal and parietal gray matter in chronic insomnia: a voxel-based morphometric study. Biol Psychiatry 2010;67:182-5.

16. Ashburner J, Friston KJ. Unified segmentation. Neuroimage 2005;26:839-51.
17. Stoffers D, Moens S, Benjamins J, van Tol MJ, Penninx BW, Veltman DJ, et al. Orbitofrontal gray matter relates to early morning awakening: a neural correlate of insomnia complaints? Front Neurol 2012;3:105.

18. Winkelman JW, Benson KL, Buxton OM, Lyoo IK, Yoon S, O'Connor S, et al. Lack of hippocampal volume differences in primary insomnia and good sleeper controls: an MRI volumetric study at 3 Tesla. Sleep Med 2010;11:576-82.

19. Fischl B. FreeSurfer. Neuroimage 2012;62:774-81.

20. Good CD, Johnsrude IS, Ashburner J, Henson RN, Friston KJ, Frackowiak RS. A voxel-based morphometric study of ageing in 465 normal adult human brains. Neuroimage 2001;14:21-36.

21. Ermer E, Cope LM, Nyalakanti PK, Calhoun VD, Kiehl KA. Aberrant paralimbic gray matter in criminal psychopathy. J Abnorm Psychol 2012;121:649-58.

22. Evans AC. Networks of anatomical covariance. Neuroimage 2013;80:489-504.

23. Alexander-Bloch AF, Vertes PE, Stidd R, Lalonde F, Clasen $\mathrm{L}$, Rapoport J, et al. The anatomical distance of functional connections predicts brain network topology in health and schizophrenia. Cereb Cortex 2013;23:127-38.

24. Bernhardt BC, Chen Z, He Y, Evans AC, Bernasconi N. Graph-theoretical analysis reveals disrupted small-world organization of cortical thickness correlation networks in temporal lobe epilepsy. Cereb Cortex 2011;21:2147-57.

25. Lerch JP, Worsley K, Shaw WP, Greenstein DK, Lenroot RK, Giedd J, et al. Mapping anatomical correlations across cerebral cortex (MACACC) using cortical thickness from MRI. Neuroimage 2006;31:993-1003.

26. Raznahan A, Lerch JP, Lee N, Greenstein D, Wallace GL, Stockman M, et al. Patterns of coordinated anatomical change in human cortical development: a longitudinal neuroimaging study of maturational coupling. Neuron 2011; 72:873-84.

27. Chen ZJ, He Y, Rosa-Neto P, Gong G, Evans AC. Age-related alterations in the modular organization of structural cortical network by using cortical thickness from MRI. Neuroimage 2011;56:235-45.

28. Spreng RN, Turner GR. Structural covariance of the default network in healthy and pathological aging. J Neurosci 2013;33:15226-34.

29. Fortier-Brochu E, Beaulieu-Bonneau S, Ivers H, Morin CM. Insomnia and daytime cognitive performance: a meta-analysis. Sleep Med Rev 2012;16:83-94.

30. Oosterman JM, van Someren EJ, Vogels RL, Van Harten B, Scherder EJ. Fragmentation of the rest-activity rhythm 
correlates with age-related cognitive deficits. J Sleep Res 2009;18:129-35.

31. Hogan RE, Wang L, Bertrand ME, Willmore LJ, Bucholz $\mathrm{RD}$, Nassif AS, et al. MRI-based high-dimensional hippocampal mapping in mesial temporal lobe epilepsy. Brain 2004;127:1731-40.

32. Joseph J, Warton C, Jacobson SW, Jacobson JL, Molteno $C D$, Eicher A, et al. Three-dimensional surface deforma- tion-based shape analysis of hippocampus and caudate nucleus in children with fetal alcohol spectrum disorders. Hum Brain Mapp 2014;35:659-72.

33. Zierhut KC, Grabmann R, Kaufmann J, Steiner J, Bogerts $B$, Schiltz K. Hippocampal CA1 deformity is related to symptom severity and antipsychotic dosage in schizophrenia. Brain 2013;136:804-14. 